\title{
Quantum-Phase Transitions of Interacting Bosons and the Supersolid Phase
}

\author{
Anne van Otterlo ${ }^{1}$, Karl-Heinz Wagenblast ${ }^{2}$, Reinhard Baltin ${ }^{2}$, \\ C. Bruder ${ }^{2}$, Rosario Fazio ${ }^{2}$, and Gerd Schön ${ }^{2}$ \\ ${ }^{1}$ Theoretische Physik, ETH-Hönggerberg, CH-8093 Zürich, Switzerland \\ ${ }^{2}$ Institut für Theoretische Festkörperphysik, Universität Karlsruhe, D-76128 Karlsruhe, FRG
}

\begin{abstract}
We investigate the properties of strongly interacting bosons in two dimensions at zero temperature using mean-field theory, a variational Ansatz for the ground state wave function, and Monte Carlo methods. With on-site and short-range interactions a rich phase diagram is obtained. Apart from the homogeneous superfluid and Mott-insulating phases, inhomogeneous chargedensity wave phases appear, that are stabilized by the finite-range interaction. Furthermore, our analysis demonstrates the existence of a supersolid phase, in which both long-range order (related to the charge-density wave) and offdiagonal long-range order coexist. We also obtain the critical exponents for the various phase transitions.
\end{abstract}

PACS numbers: 67.90.+z, 05.30.Jp

Typeset using REVTEX 


\section{INTRODUCTION}

In recent years strongly interacting bosons attracted a lot of attention. Experiments on thin granular films show a zero temperature superconductor to insulator (SI) phase transition as a function of the film thickness [1] or magnetic field [2], and experiments on Josephson junction arrays (JJA) exhibit the same SI transition as a function of the ratio between Josephson coupling and charging energy [3]. These findings are interpreted in terms of a bosonic model [4] in which the competition between the hopping of Cooper pairs and the Coulomb interaction between them is responsible for the SI transition. In relation to the effect of disorder on the SI transition a Bose-glass phase is discussed in Refs. [6] 8].

An interesting extension of the minimal model with on-site interaction only is to account for finite-range interactions, which are present in Josephson junction arrays and ${ }^{4} \mathrm{He}$-films on substrates in two dimensions, and bulk ${ }^{4} \mathrm{He}$ in three dimensions. Artificially fabricated JJA's are ideal model systems to study the superconductor to Mott-insulator transition as a function of the coupling constants or magnetic field [3]. The finite range of the interaction in these systems leads to commensurability and frustration effects. Furthermore, coexistence phases may appear that are called supersolids. In these phases a charge-density wave (CDW) that is stabilized by the interaction may coexist with superfluidity. In other words the system has diagonal long-range order (LRO) and off-diagonal LRO at the same time.

The supersolid phase was studied in the early seventies after Andreev and Lifshitz [9] suggested that vacancies in a quantum crystal such as ${ }^{4} \mathrm{He}$ might Bose-Einstein condense at low temperatures without destroying the crystal structure, thereby establishing a superfluid solid [10], or supersolid. Normally bosons at zero temperature are either superfluid (with off-diagonal LRO) or solid (with diagonal LRO). However, for a finite range of the interactions between the bosons a coexistence phase was predicted within mean-field approximations [11 [14]. Experiments have been performed on ${ }^{4} \mathrm{He}$, but no positive identification of this coexistence phase has yet been made. There are, however, hints towards such a phase [15,16. 
Recently several other kinds of coexistence phases were studied. The possibility of a spontaneous vortex anti-vortex lattice in superfluid films was explored in Ref. [17] and a coexistence phase of superfluidity and hexatic orientational order was proposed in Ref. [18. Orientational order in incompressible quantum Hall fluids is discussed in Ref. [19]. Collinear supersolids were studied in Refs. [20,21]. Finally, we mention the relation between 2D bosons and 3D flux-lines in type II superconductors (high $T_{c}$ materials) in a magnetic field [22 24]. Also in these systems different kinds of LRO may coexist and the equivalent of the supersolid is discussed in Refs. 25,26]. Related is the question whether or not vortices may form a disentangled liquid, which would imply a normal ground state for bosons with long-range Coulomb interaction [24].

In this paper both the SI transition and the supersolid phase will be studied in some detail. In the next section several related models for interacting bosons will be discussed, of which the Bose-Hubbard model is the most general. In the limit of infinite on-site interaction the Bose-Hubbard model reduces to the spin $\frac{1}{2}$ XXZ model. For a large number of bosons per lattice site the Quantum-Phase model is applicable. The phase diagram of the latter two models are first determined within mean-field theory in Section [II]. A variational Ansatz for the ground state wave function that treats all three models on equal footing [27] is presented in Section $[\mathrm{V}$. In Section $\mathrm{V}$ more accurate results for the phase diagram will be obtained by means of Quantum Monte Carlo. Part of the data has already been published as Ref. [28]. Finally the paper closes with a discussion of the various results in Section VI.

\section{THREE MODEL HAMILTONIANS}

A convenient starting point for the description of interacting lattice bosons is the BoseHubbard Hamiltonian

$$
H_{B H}=\frac{1}{2} \sum_{i j} n_{i} U_{i j} n_{j}-\mu \sum_{i} n_{i}-\frac{t}{2} \sum_{\langle i j\rangle}\left(b_{i}^{\dagger} b_{j}+b_{j}^{\dagger} b_{i}\right),
$$

where $b^{\dagger}, b$ are the creation and annihilation operators for bosons that satisfy the commu-

tation relation $\left[b_{i}, b_{j}^{\dagger}\right]=\delta_{i j}$ and $n_{i}=b_{i}^{\dagger} b_{i}$ is the number operator. The first term describes 
the density-density interaction between the bosons. We will take it to be short range, $U_{0}$ for on-site, $U_{1}$ for nearest neighbors, and $U_{2}$ for second-nearest neighbors. The second term describes the coupling of the density to a chemical potential, and the third describes the hopping from site to site with hopping integral $t$. The first two terms will cause the bosons to be localized if the interaction dominates over the hopping. In the opposite case where the hopping dominates the bosons will form a superfluid.

In the limit of large on-site interaction $U_{0}$, only the states with 0 and 1 boson per lattice site survive, and by identifying $b^{\dagger}=S^{+}=S^{x}+i S^{y}$ and $n=S^{z}+\frac{1}{2}$ the mapping to the spin $\frac{1}{2}$ XXZ Hamiltonian is obtained. It reads

$$
H_{X X Z}=\frac{1}{2} \sum_{i, j} S_{i}^{z} U_{i j} S_{j}^{z}-h \sum_{i} S_{i}^{z}-t \sum_{\langle i j\rangle}\left[S_{i}^{x} S_{j}^{x}+S_{i}^{y} S_{j}^{y}\right]
$$

where $h=\mu-\frac{1}{2} \sum_{i} U_{0 i}$. This Hamiltonian has been investigated extensively on the meanfield level for nearest-neighbor as well as next nearest-neighbor interactions.

In the limit of a large number of bosons per lattice site one may parameterize $b^{\dagger}=\sqrt{\rho} e^{i \varphi}$ and take $\rho$ equal to the boson density. Thus, only the phase remains as a dynamic variable and the Quantum-Phase Hamiltonian reads

$$
H_{Q P}=\frac{1}{2} \sum_{i, j} n_{i} U_{i j} n_{j}-\mu \sum_{i} n_{i}-J \sum_{\langle i j\rangle} \cos \left(\varphi_{i}-\varphi_{j}\right),
$$

where $J=\rho t$. The phase and number are canonical conjugate variables and satisfy $\left[\varphi_{i}, n_{j}\right]=$ $i \delta_{i j}$. This is exactly the Hamiltonian for Josephson junction arrays with an applied gate voltage in order to tune the chemical potential [29]. It is convenient to introduce a parameter $n_{0}=\mu / \sum_{i} U_{i j}$, which allows us to rewrite the Coulomb interaction and chemical potential term in Eqs. (1) and (3) as

$$
H_{\text {int }}=\frac{1}{2} \sum_{i, j}\left(n_{i}-n_{0}\right) U_{i j}\left(n_{j}-n_{0}\right) .
$$

We expect that the properties of the Quantum-Phase model will be periodic in the variable $n_{0}$ with period 1. 


\section{MEAN-FIELD}

A first insight into the properties of these models may be gained by considering their mean-field solutions. The mean-field approximation for the spin model in Eq. (2) is straightforward and was carried out by various authors [11 [13]. The question of a supersolid phase in this model was first addressed in Ref. [12] and investigated further in Ref. [13]. We review the spin mean-field theory in Subsect. IIIA. A mean-field approximation for the QuantumPhase model which is able to identify both diagonal LRO and off-diagonal LRO appeared in Ref. [14]. It is reviewed in Subsect. [IIB. Mean-field approaches to the Bose-Hubbard Model have been discussed in Refs. [6,30], but to our present knowledge there exists no

mean-field approximation for the Bose-Hubbard model which allows for the identification of a supersolid phase.

In Sect. IV we present a variational Ansatz for the many-body wave function that allows the three models to be treated on an equal footing. This is important, as it allows for a comparison between the models on the same level of approximation. Since we use a factorizable Ansatz for the wave function, it also has mean-field character.

The validity of the mean-field approximation in two dimensions is often limited, since the lower critical dimension for models with continuous symmetries is two, which is the dimension we are particularly interested in. This is a serious problem at finite temperature where the models do not even exhibit superfluid long-range order as formulated in the theorem of Mermin and Wagner, and Hohenberg [31]. Useful results may, however, be gained from mean-field approximations at zero temperature where quantum dynamics formally adds an extra dimension, the imaginary time axis. The systems then do have long-range order and mean-field results may be qualitatively correct. This will be shown in the present article by scaling arguments and confirmed by the Monte Carlo simulations. In the remainder of this section we present the different mean-field type approaches. 


\section{A. Spin Mean-Field}

The spin mean-field theory can be formulated by linearizing the spin-spin interaction. This yields the standard self-consistency equations for the magnetization

$$
\left\langle\mathbf{S}_{i}\right\rangle=-\frac{1}{2} \frac{\mathbf{H}_{i}}{\left|\mathbf{H}_{i}\right|} \tanh \left(\frac{\beta}{2}\left|\mathbf{H}_{i}\right|\right),
$$

where the subscript $i$ refers to the sublattice and $\beta$ is the inverse temperature (we use units in which $k_{B}=\hbar=c=1$ ). In order to identify spatially varying solutions one needs to introduce several sublattices, at least three for interactions including nearest and next-nearest neighbors. The symmetry in the $x y$-plane reduces the number of independent variables and most of the phase boundaries can be determined analytically. If we allow for three different sublattices $(A, B$ and $C$ ), the effective field is given by

$$
\mathbf{H}_{A}=\left(-4 t\left\langle S_{B}^{x}\right\rangle,-4 t\left\langle S_{B}^{y}\right\rangle, 2 U_{1}\left\langle S_{B}^{z}\right\rangle+2 U_{2}\left\langle S_{C}^{z}\right\rangle-h\right),
$$

and corresponding equations for $\mathbf{H}_{B}$ and $\mathbf{H}_{C}$.

The connection to hard-core bosons is made by remembering that $S^{z}$ corresponds to the particle number, and $S^{x / y}$ to the boson creation operators. A staggered magnetization in $z$ direction implies a solid ( charge-density wave) ordering of the bosons. Finite magnetization in $x y$-direction translates into off-diagonal LRO for the boson model. The coexistence phase of both types of LRO is the supersolid phase for the bosons. The result is shown in Fig. 1 1 for $U_{2}=0.1 U_{1}$. Two different types of solid ordering occur for different chemical potential, i.e., $(\pi, \pi)$ ordering around half-filling, and additional $(\pi, 0)$ and $(0, \pi)$ ordering around quarter filling.

\section{B. Quantum-Phase Model}

Now we turn to the mean-field treatment of soft-core bosons following Refs. [14, 32. After decoupling the hopping and the interactions between the different sites in Eq. (B)), one arrives at a local problem 


$$
H_{m f}^{i}\left|\psi_{i}\right\rangle=E_{i}\left|\psi_{i}\right\rangle
$$

with the $2 \pi$-periodic solutions for the wave function in the phase representation

$$
\psi_{i}\left(\varphi_{i}\right)=\exp \left(i \eta_{i} x_{i}\right) f_{i}\left(x_{i}\right)
$$

where $\varphi_{i}=2 x_{i}, \eta_{i}=2 n_{0}-\frac{2}{U_{0}} \sum_{j \neq i} U_{i j}\left(\left\langle n_{j}\right\rangle-n_{0}\right)$. After these substitutions the following Mathieu equation for $f$ is obtained

$$
\begin{gathered}
\left\{\frac{\partial^{2}}{\partial x_{i}^{2}}+e_{i}+2 r_{i} \cos \left(2 x_{i}\right)\right\} f_{i}\left(x_{i}\right)=0 \\
\text { with } \quad r_{i}=\frac{4 J}{U_{0}} \sum_{\beta}\left\langle\cos \left(\varphi_{i+\beta}\right)\right\rangle .
\end{gathered}
$$

The sum runs over nearest neighbors $\beta$ of $i$. The parameter $e_{i}$ is proportional to the energy and should be minimized. The ground state is defined via the self-consistency equation

$$
\begin{aligned}
\left\langle n_{i}\right\rangle & =\left\langle\psi_{i}^{0}\left|n_{i}\right| \psi_{i}^{0}\right\rangle \\
\left\langle\cos \left(\varphi_{i}\right)\right\rangle & =\left\langle\psi_{i}^{0}\left|\cos \left(\varphi_{i}\right)\right| \psi_{i}^{0}\right\rangle
\end{aligned}
$$

In addition the free energy has to be minimized in order to have an unambiguous solution of Eq. (11). The numerical solution of this set of equations is straightforward. In order to allow for spatially varying solutions we again define sublattices. Our numerical solutions confirm the results of Ref. [14], however, some details differ.

For on-site interaction we obtain Mott insulating lobes centered around integer values $N$ of $n_{0}$, see Fig. 2. In these incompressible lobes the density is pinned to $N$. At half integer values of $n_{0}$ the critical value of the hopping vanishes, due to the degeneracy in energy of states with $N$ and $N+1$ particles per site.

Including nearest-neighbor interaction the calculation has to be performed on two sublattices. We obtain two different kinds of insulating lobes, one with integer filling and homogeneous density, another with half-integer filling and a checkerboard charge-density wave, or solid order, centered around half integer values of $n_{0}$. In addition we find a region 
of supersolid phase around the half lobe where the checkerboard charge-density wave and

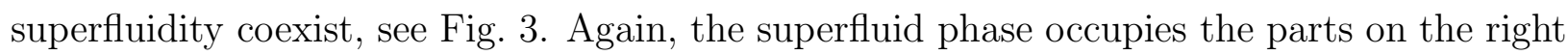
of the phase diagram and also between the insulating lobes down to zero hopping, in slight contrast to the result of Ref. [14].

The fraction of supersolid phase in the phase diagram depends on the ratio $U_{1} / U_{0}$, see Fig. . In the hard-core limit $U_{0} \rightarrow \infty$ the supersolid phase vanishes. An expansion of the ground state wave function $\left|\psi_{i}^{0}\right\rangle=\sum_{n} c_{n}^{i}|n\rangle$ into particle number eigenstates $|n\rangle$ yields more

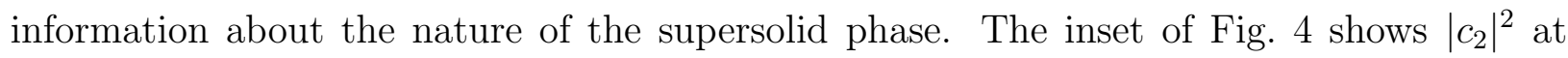
$n_{0}=0.5$ as a function of $J / U_{0}$. The figure indicates that double occupancy is important for the supersolid phase. Thus, we conclude that the supersolid phase is favored by fluctuations in the particle number.

The inclusion of further interactions leads to more structure in the phase diagram. For next-nearest neighbor interaction we introduce three sublattices. As a result, The phase diagram shows a lobe with quarter filling and a phase where this quarter structure and superconductivity coexist, see Fig. 5.

\section{Hard-core vs. Soft-core}

The previous subsections showed two extreme cases of the Bose-Hubbard model, the hard-core limit and the limit of large particle numbers. Although the phase diagrams look similar, these two limits behave qualitatively different as far as the supersolid phase is concerned.

In the hard-core limit the existence of the supersolid is related to a finite next-nearest neighbor interaction. The supersolid does not exist in the model including nearest-neighbor interaction only [13]. Furthermore, there is no supersolid phase at exactly half filling. This leads to the following interpretation of the supersolid phase. At densities corresponding to half filling the particles form an incompressible solid. Away from half filling superfluidity is enabled by defects in the solid structure that Bose-Einstein condense. This interpreta- 
tion does, however, not explain why next-nearest neighbor interaction is necessary for the formation of the supersolid.

In the limit of many particles per site the Quantum-Phase model applies. In this case the supersolid phase already exists for nearest-neighbor interaction only and at half filling. This is related to excitations which are forbidden in the hard-core limit. We observe that a large nearest-neighbor interaction $U_{1}$ or small on-site interaction $U_{0}$ favors the supersolid, in the hard-core limit the supersolid is suppressed. In the soft-core limit the supersolid phase is also present at half-filling at the tip of the checkerboard lobe. Thus, it seems that the system itself generates the defects (particle-hole excitations) that Bose condense, thereby turning the solid into the supersolid.

\section{VARIATIONAL METHOD}

Here we present a method which allows us to treat the three models within one scheme [27]. The idea is to guess a variational wave function for the problem [33]. We use a variational wave function that is inspired by earlier successful Gutzwiller-like treatments of the spin model [34] and the Bose-Hubbard model [35, 36]. The occupation number representation is well suited for all three models. Explicitly we resolve the identity as

$$
1=\sum_{\left\{n_{i}\right\}}\left|\left\{n_{i}\right\}\right\rangle\left\langle\left\{n_{i}\right\}\right|
$$

and express the trace as

$$
\operatorname{Tr} \ldots=\sum_{\left\{n_{i}\right\}}\left\langle\left\{n_{i}\right\}|\ldots|\left\{n_{i}\right\}\right\rangle
$$

where the $n_{i}$ are in $[0,1]$ for the spin $\frac{1}{2} \mathrm{XXZ}$ model, in $[0 . . \infty]$ for the Bose-Hubbard $(\mathrm{BH})$

model, and in $[-\infty . \infty]$ for the Quantum-Phase (QP) model. Besides the difference in the allowed values of $n_{i}$, the matrix elements differ for the three models, e.g.,

$$
\begin{array}{ll}
\text { BH model: } & \left\langle\left\{n_{i}^{\prime}\right\}\left|b_{k}\right|\left\{n_{i}\right\}\right\rangle=\left\langle\left\{n_{i \neq k}^{\prime}\right\}\right|\left\{n_{i \neq k}\right\rangle\left\langle n_{k}^{\prime} \mid n_{k}-1\right\rangle \sqrt{n_{k}} \\
\text { QP model: } & \left\langle\left\{n_{i}^{\prime}\right\}\left|\exp i \varphi_{k}\right|\left\{n_{i}\right\}\right\rangle=\left\langle\left\{n_{i \neq k}^{\prime}\right\}\right|\left\{n_{i \neq k}\right\rangle\left\langle n_{k}^{\prime} \mid n_{k}-1\right\rangle
\end{array}
$$


As our variational wave function we take the product of single-site wave functions. This amounts to neglecting certain correlations and is therefore similar to a mean-field approximation. We use the following Ansatz

$$
\begin{aligned}
& |\psi\rangle=\prod_{i=1}^{N} \frac{1}{\sqrt{Z_{i}}} \sum_{\left\{n_{i}\right\}} \exp \left\{-k_{i}\left(n_{i}-m_{i}\right)^{2} / 2\right\}\left|n_{i}\right\rangle \\
& \text { where } \quad Z_{i}=\sum_{\left\{n_{i}\right\}} \exp \left\{-k_{i}\left(n_{i}-m_{i}\right)^{2}\right\},
\end{aligned}
$$

and $k_{i}$ and $m_{i}$ are real variational parameters. The single-site wave function is a superposition of boson number eigenstates weighted with a Gaussian. The average is controlled by $m_{i}$, the width by $k_{i}$, and spatial variations (different sublattices $i$ ) are allowed. By minimizing the energy expectation value $E=\langle\psi|H| \psi\rangle$ with respect to $k_{i}$ and $m_{i}$ we obtain the ground state wave function within our approximation. In the following, expectation values are understood to be calculated with the ground state wave function. As the discrete sums can in general not be performed analytically, we treat the problem numerically. For on-site and nearest-neighbor interactions two sublattices arranged in a checkerboard configuration are introduced. We define the superconducting order parameter as $\langle\exp (i \varphi)\rangle$ for the $\mathrm{QP}$ model, $\langle b\rangle$ for the BH model, and $\left\langle S^{-}\right\rangle$for the XXZ model. The structure factor

$$
S(\vec{q})=\left(\frac{1}{N}\right)^{2} \sum_{i, j} \exp \left[i \vec{q} \cdot\left(\vec{r}_{i}-\vec{r}_{j}\right)\right]\left\langle n_{i} n_{j}\right\rangle
$$

yields information about the solid order. A non-vanishing structure factor at finite $\vec{q}$ signals diagonal LRO. By decomposing the lattice into two sublattices we gain information about $S(\pi, \pi)$. A finite $S(\pi, \pi)$ corresponds to a checkerboard arrangement of the particles. For next-nearest neighbor interactions we introduce four sublattices which yields additional information about $S(\pi, 0)$ and $S(0, \pi)$.

Figure 6 shows the phase diagram for the QP model with on-site and nearest-neighbor interaction obtained with our variational Ansatz. Both the superfluid-insulator and the crystalline order transition can be identified and agree with previous mean-field calculations [14,28]. The phase boundaries are periodic in $n_{0}$. We find that superfluidity sets in 
simultaneously on both sublattices $A$ and $B$. Within the supersolid phase the value of the superconducting order parameter on $A$ differs from that on $B$, but both are nonzero. Beyond the crystalline order phase boundary the order parameter does not exhibit any difference on the sublattices.

For the XXZ model the variational method confirms that there is no supersolid for on-site and nearest-neighbor interaction only. In the presence of next-nearest neighbor interaction we find supersolid phases in perfect agreement with [13] and Fig. 1.

The phase diagram for the $\mathrm{BH}$ Hamiltonian is shown in Fig. 7. The size of the lobes decreases with increasing $n_{0}$. At point $\alpha$ the supersolid vanishes. This might be due to the lower bound for the occupation numbers $n \geq 0$. For small $n_{0}$ charge fluctuations are suppressed. Hence, we conclude that charge fluctuations are necessary for the supersolid. For large $n_{0}$ the phase boundaries of the QP model approach those of the $\mathrm{BH}$ model, if the hopping is rescaled according to Section प1], i.e., if $t$ and $J / n_{0}$ are identified.

\section{QUANTUM MONTE CARLO}

Several methods are available for determining the phase diagram for the three model Hamiltonians by numerical means. Exact diagonalization is possible for the XXZ model and small systems, i.e., less than about 26 lattice sites. Furthermore it is possible to perform Monte Carlo simulations for all the three models on much larger systems. The Bose-Hubbard model was studied in one dimension in Refs. [37, 38], and one data point for the phase boundary was obtained in two dimensions in Ref. [39]. The Bose-Hubbard model was more recently studied in the context of a supersolid phase in Ref. [20]. The two-dimensional Quantum-Phase model was studied in Ref. [40] in relation to the universal conductivity at the SI transition and in Refs. [21,28] in relation to supersolid phases.

In this section we present more results on Monte Carlo simulations of the two-dimensional QP model in addition to those in Ref. [28]. We discuss the on-site problem and compare to the analytic results of $t / U$ expansions [ [41,42]. The inclusion of nearest-neighbor interactions 
allows us to study the supersolid phase.

\section{A. Duality Transformation}

In order to study the Quantum-Phase model by means of Monte Carlo we map the 2D quantum model onto a $(2+1) \mathrm{D}$ classical model of discrete divergence-free currents. The

essential feature of this mapping were presented in Ref. [23 and the derivation makes use of duality transformations that were developed in Ref. [43]. We start from the basic expression for the partition function $Z$ for the Quantum-Phase model of Eq. (3)

$$
Z=\operatorname{Tr} \exp \left(-\beta H_{Q P}\right)
$$

where $\beta$ is the inverse temperature. We go over to a Euclidean path-integral formulation by introducing time-slices, i.e., dividing $\beta$ in $N_{\tau}$ intervals of size $\epsilon$, such that $N_{\tau} \epsilon=\beta$. Inserting complete sets of states at each time slice we arrive at

$$
\begin{aligned}
Z= & \sum_{\left\{n_{i, \tau}=0, \pm 1, \pm 2, \cdots\right\}} \int \mathcal{D} \varphi_{i, \tau} \exp \left\{-\frac{\epsilon}{2} \sum_{i j, \tau} n_{i, \tau} U_{i j} n_{j, \tau}+\epsilon \mu \sum_{i, \tau} n_{i, \tau}\right. \\
& \left.+i \sum_{i, \tau} n_{i, \tau} \dot{\varphi}_{i, \tau}+\epsilon J \sum_{\langle i j\rangle, \tau} \cos \left(\varphi_{i, \tau}-\varphi_{j, \tau}-A_{i j}\right)\right\} .
\end{aligned}
$$

We included a coupling to a vector potential $A_{i j}=\left(2 \pi / \Phi_{0}\right) \int_{i}^{j} \mathbf{A} \cdot d \mathbf{l}$ for later convenience. In order to proceed, we make one approximation, the Villain approximation 44 for the cosine term. This amounts to expanding the cosine around all its minima

$$
\exp \left\{\epsilon J \cos \left(\varphi_{i \tau}-\varphi_{j \tau}-A_{i j}\right)\right\} \approx \sum_{\left\{m_{i j, \tau}\right\}} \exp \left\{-\frac{\epsilon J F(\epsilon J)}{2}\left(\varphi_{i \tau}-\varphi_{j \tau}-2 \pi m_{i j, \tau}-A_{i j}\right)^{2}\right\}
$$

where $m$ is a directed discrete field that lives on the bonds between lattice sites. The function $F$ is determined by demanding that the first two Fourier coefficients of the expressions on the left and right hand side of Eq. (19) are equal [44] and is given by

$$
F(x)=\frac{1}{2 x \log \left\{I_{0}(x) / I_{1}(x)\right\}}
$$


For large $\operatorname{arguments} F$ approaches 1, as is clear from a direct expansion of the cosine potential. After a subsequent Poisson resummation, i.e., writing

$$
\sum_{\left\{m_{i j, \tau}\right\}} f\left[m_{i j, \tau}\right]=\sum_{\left\{\mathcal{J}_{i j, \tau}\right\}} \int \mathcal{D} m f\left[m_{i j, \tau}\right] \exp \left(2 \pi i \sum_{i j, \tau} m_{i j, \tau} \mathcal{J}_{i j, \tau}\right),
$$

an integration over the fields $m_{i j, \tau}$ and the phases $\varphi_{i, \tau}$ yields a representation in terms of divergence-free discrete current loops

$$
\begin{gathered}
Z=[\epsilon J F(\epsilon J)]^{-1} \sum_{\left\{\mathcal{J}_{i, \tau}^{\nu}\right\}} \delta\left(\nabla_{\nu} \mathcal{J}^{\nu}\right) \exp \{-S[\mathcal{J}]\}, \\
S[\mathcal{J}]=\frac{\epsilon}{2} \sum_{i j, \tau} \mathcal{J}_{i, \tau}^{\tau} U_{i j} \mathcal{J}_{j, \tau}^{\tau}-\epsilon \mu \sum_{i, \tau} \mathcal{J}_{i, \tau}^{\tau}+\frac{1}{2 \epsilon J F(\epsilon J)} \sum_{i, \tau, a=x, y}\left(\mathcal{J}_{i, \tau}^{a}\right)^{2}-i \sum_{i, \tau} \vec{A}_{i} \cdot \overrightarrow{\mathcal{J}}_{i, \tau} \\
=\sqrt{\frac{2}{K}}\left\{\sum_{i j \tau}\left(\mathcal{J}_{i, \tau}^{\tau}-n_{0}\right)\left(\delta_{i j}+\frac{U_{1}}{U_{0}} \delta_{\langle i j\rangle}\right)\left(\mathcal{J}_{j, \tau}^{\tau}-n_{0}\right)+\sum_{i \tau, a=x, y}\left(\mathcal{J}_{i, \tau}^{a}\right)^{2}\right\}-i \sum_{i, \tau} \vec{A}_{i} \cdot \overrightarrow{\mathcal{J}}_{i, \tau},
\end{gathered}
$$

where $\nu=x, y, \tau$. The effective coupling constant is given by $\sqrt{2 / K}$ with $K=8 F(\epsilon J) J / U_{0}$, where $\epsilon$ is defined implicitely by $\epsilon=1 / \sqrt{U_{0} F(\epsilon J) J}$. The time components $\mathcal{J}_{i, \tau}^{\tau}$ of the current correspond to the boson numbers $n_{i, \tau}$ along their world lines. We keep track of the energy dependence of the determinant that arises from integrating out the $m_{i j, \tau}$. This is relevant for determining the energy density and specific heat by means of Monte Carlo. For zero magnetic field $(\vec{A}=0)$ the action in the representation of Eq. (21) is real, and therefore suitable for Monte Carlo methods. Using the standard Metropolis algorithm we generate configurations of currents in a system of size $L \times L \times L_{\tau}$. The condition that the currents $\mathcal{J}^{\nu}$ be divergence-free is taken into account by making Monte Carlo steps that preserve the property $\nabla_{\nu} \mathcal{J}^{\nu}=0$. Thus, we create or annihilate small current loops at every lattice site in all three directions, as well as periodic current loops that go through the whole system which is taken to have periodic boundary conditions in all three directions. The generation of configurations may be done in a canonical as well as in a grand-canonical way. Here we work in the grand-canonical ensemble in order to make contact to the mean-field phase diagrams.

In the last line of Eq. (21) we used $\epsilon=1 / \sqrt{U_{0} F(\epsilon J) J}$. With this choice the couplings are isotropic for on-site interaction, leading to an efficient numerical algorithm. The choice 
of the time lattice spacing $\epsilon$ needs some justification. Introducing time slices in the partition function Eq. (18) is exact in the limit $\epsilon \rightarrow 0$. This would produce extremely anisotropic couplings in the current-loop model and the numerical simulation would become impossible. Thus, a more reasonable choice for the time lattice spacing is necessary. The plasma

frequency $\omega_{p}=\sqrt{J U_{0}}$ is a natural frequency for spin waves. A cutoff beyond this frequency by introducing a time spacing $\epsilon \approx 1 / \omega_{p}$ should not do any harm. We tested the dependence on the choice of $\epsilon$ for the quantities of interest and observed that they are not sensitive to variations of $\epsilon$ by one order of magnitude, provided that the temperature is kept constant, i.e., $\beta=1 / T=$ const. $=N_{\tau} \epsilon$. Additional justification for the choice of a finite time spacing $\epsilon$ in the present paper is given by the fact that we are only interested in the critical regime, where a high-frequency cutoff should be irrelevant.

\section{B. Finite-Size Scaling}

The mapping to the current-loop model Eq. (21) is well suited for simulating the behavior of the Quantum-Phase model with short range interactions. In practice we are able to study system sizes up to $12 \times 12 \times 12$, or $10 \times 10 \times 25$, respectively. The factor that limits the largest systems to have $L=12$ is the necessity to make the periodic current loops in the spatial directions. Only they can change the superfluid stiffness, which is a topological quantity that cannot be changed by making local current loops. As phase transitions take place only in the thermodynamic limit, we need an additional ingredient that relates the data obtained on finite-size systems to infinite system size properties as the critical coupling and exponents. This is provided by finite-size scaling.

Let us first consider the onset of superfluidity in the system. Therefore we study the behavior of the superfluid stiffness $\rho_{0}$, a quantity which measures the response to a twist in boundary conditions for the phase. A finite value of $\rho_{0}$ in the thermodynamic limit reflects long-range phase coherence, i.e., off-diagonal LRO or superfluidity. A twist in the boundary conditions for the phases by an angle $\Theta$ increases the free energy density by 


$$
\Delta f=\frac{1}{2} \rho_{0}\left(\frac{\Theta}{L}\right)^{2}
$$

The twist may be realized by applying a magnetic field. In general the frequency and wave-vector dependent stiffness $\rho\left(k, \omega_{\mu}\right)$ can be defined as

$$
\rho\left(k, \omega_{\mu}\right)=\left.\left(\frac{1}{2 e}\right)^{2} \sum_{i, \tau} \frac{-\delta^{2} \ln Z}{\delta A_{i, \tau}^{x} \delta A_{0}^{x}}\right|_{\vec{A}=0} e^{-i \omega_{\nu} \tau-i k r_{i}} .
$$

The zero-frequency and zero wave-vector component of $\rho\left(k, \omega_{\mu}\right)$ defines the superfluid stiffness. In terms of the currents it reads

$$
\rho_{0}=\rho_{\left(k=0, \omega_{\mu}=0\right)}=\frac{1}{L^{d} L_{\tau}}\left\langle\left(\sum_{i, \tau} \mathcal{J}_{i, \tau}^{x}\right)^{2}\right\rangle=\frac{1}{L^{d-2} L_{\tau}}\left\langle w_{x}^{2}\right\rangle .
$$

The winding numbers $w_{x}=\frac{1}{L} \sum_{i, \tau} \mathcal{J}_{i, \tau}^{x}$ can be measured easily; they are only modified by periodic current loops, local loops do not change their value. The symmetry in $x / y$-direction further simplifies the numerics.

Direct measurement in the critical regime is ruled out by the divergence of the correlation length $\xi$ at the continuous transition. The powerful method of finite-size scaling, however, takes advantage of this fact and allows us to study the critical behavior. Near a continuous phase transition the diverging coherence length is cut off by the finite system size $L$. As a result all quantities will depend on the ratio $\xi / L$. A finite-size scaling relation for the superfluid stiffness $\rho_{0}$ is readily derived [45]. Assuming hyperscaling, the critical part of the free energy density behaves as the inverse correlation volume $\xi^{d} \xi_{\tau}$. Here, $\xi$ is the correlation length in real space which may be different from the correlation length $\xi_{\tau}$ in imaginary time, depending on the quantum dynamics of the model. The dynamical critical exponent $z$ can be introduced through $\xi_{\tau} \propto \xi^{z}$. Combining Eq. (22), the hyperscaling relation and the fact that $\xi$ is cut off by $L\left(\xi_{\tau}\right.$ by $\left.L_{\tau}\right)$ we arrive at the scaling behavior

$$
\rho_{0}=L^{2-d-z} \tilde{\rho}\left(b L^{1 / \nu} \delta, L_{\tau} / L^{z}\right)
$$

Here, $b$ is a non-universal scale factor and $\tilde{\rho}$ is a universal scaling function with a smooth dependence on $L_{\tau} / L^{z}$ and $\xi / L=\delta^{-\nu} / L$. We assumed a power law critical behavior $\xi \propto \delta^{-\nu}$ 
with dimensionless distance to the transition $\delta=\left(K-K^{*}\right) / K^{*}$ and critical coupling $K^{*}$. At the critical point $(\delta=0), L^{d+z-2} \rho_{0}$ is a function of $L_{\tau} / L^{z}$ only. Thus, plots of $L^{d+z-2} \rho_{0}$ vs. $K$ will intersect at the transition if $L_{\tau} / L^{z}$ is kept constant, with no further fitting involved. Furthermore, the data for $L^{d+z-2} \rho_{0}$ plotted as a function of $L^{1 / \nu} \delta$ for different system sizes should collapse onto one single curve. This allows the exponent $\nu$ to be determined.

As we are interested also in diagonal LRO and a possible supersolid phase, we measure the structure factor as well. The structure factor is the $(\pi, \pi)$-component of the equal time density-density correlation, see Eq. (16), and may be derived as the second variation of the free energy with respect to a staggered chemical potential. Expressed in terms of the currents $\mathcal{J}^{\tau}$ it reads

$$
S_{\pi}=\frac{1}{L^{4} L_{\tau}}\left\langle\sum_{i j, \tau}(-1)^{i+j} \mathcal{J}_{i, \tau}^{\tau} \mathcal{J}_{j, \tau}^{\tau}\right\rangle
$$

The scaling relation for the structure factor is

$$
S_{\pi}=L^{-2 \beta / \nu} \tilde{S}\left(b^{\prime} L^{1 / \nu} \delta, L_{\tau} / L^{z}\right)
$$

This scaling relation arises, since the structure factor $S_{\pi}$ is related to the square of the staggered magnetization order parameter $M=\sum_{i}(-1)^{i} \mathcal{J}_{i}^{\tau}$ for which the exponent $\beta$ is the order parameter exponent. Near the phase transition, $M \sim \delta^{\beta}$ and $\xi \sim \delta^{-\nu}$, from which the quoted form follows. From a three-parameter fit to the scaling relation for different system sizes, the exponents $\nu$ and $\beta$ as well as the critical coupling constant $K^{*}$ are determined. Again, plots of $L^{2 \beta / \nu} S_{\pi}$ will cross at the critical point if the ratio $L_{\tau} / L^{z}$ is kept constant.

In both scaling relations a knowledge of $z$ is assumed. This is well-known for the SI transition and the scaling of $\rho_{0}$, i.e., in general $z=2$, except for the tips of the lobes where $z=1$ as dictated by particle hole-symmetry. This argument is based on a coarse-graining treatment [5] that allows for the explicit construction of a Ginzburg-Landau-Wilson free energy functional for the superconducting order parameter [6,46, 48, from which $z$ can be read off. For the transition related to the structure factor the situation is less clear. We were, for instance, not yet successful in explicitly constructing the Ginzburg-Landau-Wilson free 
energy functional for the CDW transition. From symmetry arguments one expects $z=1$ at the mean-field level [49]. Also the spin-wave analysis of Ref. [20] predicts $z=1$. However, it is unclear whether or not $z$ for the CDW will be renormalized by the coupling to the gapless sound mode in the superfluid part. As a working hypothesis we take $z=1$ and comment later on the consistency of this assumption.

\section{Numerical Results}

We report about the SI transitions for on-site interaction and nearest-neighbor interaction in the complete range of the chemical potential. A phase diagram is mapped out and the scaling predictions are verified. The existence of a supersolid phase in a finite region of the phase diagram is firmly established.

The case of on-site interaction has been extensively studied in the past years. A phase diagram consisting of lobe-like structures in the $t-\mu$ or $J-n_{0}$ plane is found. Mottinsulating lobes are centered around integer values of $n_{0}$. This has been observed in meanfield approximations, $t / U$ expansions [41,42], and in Monte Carlo simulations of the onedimensional Bose Hubbard model [37. The shape of the lobes in two dimensions is yet unknown. Therefore we first map out this phase diagram which is shown in Fig. 2. We performed the simulations for fixed $n_{0}$ and tuned through the transition by varying the effective coupling $K$. In the phase diagram in Fig. 2 this corresponds to moving on horizontal lines through the phase transition.

At integer values of $n_{0}$ the system is particle-hole symmetric and we have $z=1$. According to the scaling form of Eq. (25) we use $L=L_{\tau}$ which keeps the second argument of the scaling function constant. Four different system sizes with $L=6,8,10,12$ where studied. For the largest systems we typically use $10^{6}$ sweeps through the system for equilibration and measurement. In each sweep we try all possibilities of local and periodic current loops. Without any fitting the curves of $\rho_{0} L^{d+z-2}=\rho_{0} L$ vs. $K$ should cross in the critical point $K^{*}$.

This serves as a good test of the scaling prediction. The critical exponent $\nu$ can be extracted 
by fitting the data of different system sizes near the transition to one scaling curve. This is shown in Fig. 8 where we plot the data as a function of $\delta L^{1 / \nu}$. For $n_{0}=0$ we obtain the critical coupling $K^{*}=0.886 \pm 0.003$, and using a $\chi^{2}$ fit $\nu=0.69 \pm 0.06$. The exponent $\nu$ agrees with the expected $3 \mathrm{D}-\mathrm{XY}$ behavior, where $\nu \approx 2 / 3$.

Away from the symmetry points at the tips of the lobes the dynamical critical exponent is $z=2$. In order to keep $L_{\tau} / L^{z}$ constant we simulate systems with $L_{\tau}=L^{2} / 4$, i.e., $L \times L \times L_{\tau}=6 \times 6 \times 9,8 \times 8 \times 16$ and $10 \times 10 \times 25$. Now the curves of $\rho_{0} L^{2}$ should cross in

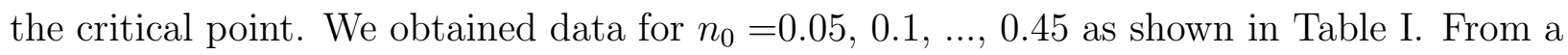
fit to the scaling form we find $0.42<\nu<0.52$ with errors of $\approx 20 \%$. This is in agreement with the expected mean-field transition where $\nu=1 / 2$. A more accurate determination of the critical exponents turns out to require an enormous numerical effort with our method and is beyond the scope of our present work.

In Fig. 2, the Monte Carlo data are shown together with mean-field phase boundaries and the results of the $t / U$-expansion of Refs. 41,42 in the limit of large $n_{0}$ (or $\mu$ ). A lobe shape is observed, these lobes are sharper than predicted by mean-field, but smoother than expected from the $t / U$-expansion. Approaching $n_{0}=0.5$ we observe that the critical value of $J / U_{0}$ decreases and a simple extrapolation yields $J^{*}=0$ for $n_{0}=0.5$. A numerical answer to the question whether $J^{*}$ is zero at $n_{0}=0.5$ is not possible, since the acceptance rates are very small for small $J / U_{0}$.

The inclusion of nearest-neighbor interactions yields a richer phase diagram. The scaling for the superfluid stiffness is not modified by the inclusion of short-range interactions, i.e., the universality class is preserved. We expect $z=1$ in the case of particle-hole symmetry, $z=2$ otherwise. For nearest-neighbor interaction the lines with integer and half-integer values of $n_{0}$ exhibit particle-hole symmetry. For the transition related to charge-density wave order, we expect $z=1$ throughout the phase diagram. Guided by the mean-field phase diagram we expect the existence of two different kinds of lobes, with homogeneous particle densities centered around integer values of $n_{0}$, and with a checkerboard charge-density wave centered around half integer values of $n_{0}$. The supersolid phase is expected in the vicinity of the 
checkerboard lobe.

For $n_{0}=0$ and 0.5 we simulated $L \times L \times L$ systems, where $L=6,8,10,12$, as suggested by particle-hole symmetry and $z=1$. For $n_{0}=0.1,0.2$ and 0.4 we obtain $z=2$ for the superfluid transition. In order to keep the ratio $L_{\tau} / L^{z}$ constant, we simulated $L \times L \times L^{2} / 4$ systems, where $L=6,8,10$. For $n_{0}=0.4$ near the transition for the diagonal LRO we performed the calculations with both $z=1$ and $z=2$. The results are summarized in Fig. 9 and Fig. 10 and Table 【1. In the following we discuss our data in detail.

First we present our data for $n_{0}=0.5$. Figure 9 shows that there are two separate transitions for diagonal and off-diagonal LRO with a coexistence region in between where both the superfluid density and the structure factor scale to a finite value in the thermodynamic limit. This demonstrates the coexistence of diagonal LRO and off diagonal LRO for soft-core bosons with nearest-neighbor interaction in 2 dimensions. Table \1 shows that the exponent $\nu$ is different for the two transitions. For the transition related to superfluidity (point $\beta$ in Fig. 3) we find $\nu=0.65 \pm 0.08$ which is consistent with the 3D XY universality class. For the transition related to crystalline order (point $\gamma$ ) the universality class is not known. We find $\nu \approx 0.55$ and $\beta \approx 0.21$.

Also at $n_{0}=0.4$ we find two separate transitions that are the boundaries for the supersolid phase in between, see Table II. As compared to $n_{0}=0.5$ both transitions are shifted to smaller values of the coupling constant $K$. This is consistent with the mean-field phase diagram. Again the two transitions have different critical exponents. The transition related to superfluidity (the line separating phases "Sol" and "SSol" in Fig. 3) has $\nu=0.44 \pm 0.08$ which is consistent with a mean-field transition in $d+z=4$ effective dimensions. For the transition related to crystalline order (between phases "SSol" and "SF") we compare the data for $z=2$ and $z=1$ in Fig. 10. A fit to the expected scaling behavior is equally possible in both cases. The statistical errors of the data for $z=2$ are larger, since the simulation for $z=2$ requires larger $L_{\tau}$ (up to 25) which decreases the acceptance ratio for periodic loops in this direction. The values for the critical coupling and the critical exponents for both $z=1$ and $z=2$ are shown in Table II, they coincide within the error bars. With the 
values of Table $\llbracket$ the scaling relation $2 \beta=\nu(d+z-2+\eta)$ leads to $0.8 \pm 0.2=z+\eta$, with correlation function exponent $\eta$ (small and usually positive). This rules out $z=2$ and one is led to conclude that indeed $z \sim 1$ for the crystalline transition, independent of $n_{0}$.

The small value of $\beta$ rules out mean-field behavior for the CDW transition. Insight into the nature of this transition is gained by the following consideration. In the neighborhood of this transition, i.e., far in the superfluid phase, the $\mathrm{x}, \mathrm{y}$-components of the currents $\mathcal{J}$ fluctuate strongly and may be integrated out as Gaussian fluctuations. In other words: one expects the staggered magnetization order parameter to couple to the gapless $4^{\text {th }}$ sound mode of the superfluid. This yields strong long-range interactions in the time direction for the $\tau$-components of the currents $\mathcal{J}$. It is likely that these long-range interactions are a relevant perturbation and suppress the exponent $\beta$.

Finally, we discuss the data for $n_{0}=0,0.1$, and 0.2 . In these cases there is only one phase transition, as the Mott-insulating lobes (phase "MI" in Fig. 3) do not have any non-trivial crystalline order. Our data are consistent with a transition in the 3D XY universality class for $n_{0}=0$ and a mean-field phase transition for $n_{0}=0.1$ and 0.2 .

\section{DISCUSSION}

We studied the $T=0$ phase diagram of two-dimensional interacting bosons by various techniques. The combination of the various aspects of these treatments allow us to draw the following conclusions.

For on-site interaction we have seen the lobe structure of the phase boundary in the $t-\mu$ or $J-n_{0}$ plane. This structure is exactly periodic in the chemical potential $n_{0}$ in the Quantum-Phase model of Eq. (3). In mean field or variational treatments of the different models, the lobes are parabolic near their tips. The Monte Carlo results, however, show that the lobes are sharper than predicted by mean-field approximations. This deviation near the tips of the lobes is expected by scaling arguments: due to the additional particlehole symmetry at the tips of the lobes, the effective dimension is reduced to $d_{\text {eff }}=d+z=3$ 
and enhanced fluctuations destroy part of the ordered (superfluid) phase. This is consistent with the value of the critical exponent $\nu$ obtained from the simulations $(\nu \approx 2 / 3)$ that agrees well with a 3D XY critical point. Similar sharpening of the lobes is seen in a higher-order $t / U$ expansion for the $\mathrm{BH}$ model [41,42].

A central point of this paper is the consideration of finite-range interactions. They lead to a richer phase diagram, due to commensurability effects. In particular, charge-density wave or solid order appears in parts of the phase diagram. In our definition the solid phase is characterized by the breaking of the translation symmetry of the underlying lattice, i.e., if a density wave with a wave vector smaller than the lattice is present.

Upon inclusion of nearest-neighbor interactions new half-filled lobes around half-integer $n_{0}$ appear in the phase diagram, in which the checkerboard $(\pi, \pi)$ density wave is stable. For next-nearest neighbor interactions additional density waves exist in which 1 or 3 out of 4 sites are occupied, around $n_{0}=1 / 4$ or $3 / 4$. These pure density waves are incompressible Mott-insulators. Surprisingly, each Mott-insulating charge-density wave seems to have a corresponding compressible supersolid phase in which the particular CDW order coexists with off diagonal LRO.

For soft-core bosons the supersolid phases appear already for nearest-neighbor interaction. Hard-core bosons, on the other hand, supersolidify only if at least next-nearest neighbor interaction is present. Another difference is the presence of a supersolid at the tip of the half lobe: hard-core bosons only form a supersolid away from half-integer filling, whereas soft-core bosons do so also at half-integer filling.

The difference between Bose-Hubbard and Quantum-Phase models is another issue. Our results indicate that, on the mean-field level, the two models are, apart from a trivial rescaling of the hopping matrix element, almost identical for large $\mu$ or $n_{0}$. For small $\mu$ corresponding to a filling of less than one boson per site the mean-field solution for the two models differ essentially and are not described by a simple rescaling of the parameters. Within our mean-field approximation we find that the supersolid phase is suppressed around the solid lobe with exactly half filling (alternating empty and singly-occupied sites). This may be one 
of the reasons why in a recent numerical simulation of the $\mathrm{BH}$ model [20] a supersolid phase was not found for exactly half filling.

Our combined mean-field and Monte Carlo analysis has shown that the density-wave order transition is not mean-field like. Both the exponents $(\beta \approx 0.21$ and $\nu \approx 0.55)$ and the location of the phase boundary deviate considerably from the mean-field results $(\beta=1 / 2$ and $\nu=1 / 2$ ), see also Fig. 3. This may be related to the coupling of the CDW order parameter to the gapless $4^{\text {th }}$ sound mode in the superfluid and remains a subject for further study. A related issue is the value of the dynamical critical exponent $z$ for this transition. The value $z=1$ used in the simulations gives good scaling. However, other values of $z$ turn out to give reasonable scaling as well and yield identical values for $\beta, \nu$ and $K^{*}$. It seems that the CDW transition is rather indifferent to the value of $z$ used to scale the systems studied numerically. From the scaling relation $2 \beta / \nu=d+z-2+\eta$ we expect the combination $z+\eta \approx 0.8 \pm 0.2$ which would indicate either a small $\eta$ and renormalized $z \approx 0.8$ or $z \approx 1$ and $\eta$ negative. Further simulations and renormalization group studies are required for solving this problem.

In summary we performed an analysis of interacting bosons in $2 \mathrm{D}$ within zero temperature lattice models, using and comparing both mean-field theory and exact Quantum Monte Carlo simulations. We obtained the full phase diagram for on-site interaction as well as for nearest-neighbor interactions, in which case our simulations establish the existence of a supersolid phase in which a CDW and superfluidity coexist.

Acknowledgments: We thank G. T. Zimanyi for motivation and discussions, and J. K. Freericks and H. Monien for discussions and their data as plotted in Fig. 2. The work was partially supported by 'Sonderforschungsbereich 195' of the DFG and by the Swiss Nationalfonds (AvO). 


\section{TABLES}

\begin{tabular}{||c|c|c|c|c||c|c|c|c|c||}
\hline \hline$n_{0}$ & $z$ & $K^{*}$ & $J^{*} / U_{0}$ & $\nu$ & $n_{0}$ & $z$ & $K^{*}$ & $J^{*} / U_{0}$ & $\nu$ \\
\hline \hline 0.00 & 1 & $0.886 \pm 0.003$ & 0.152 & $0.69 \pm 0.06$ & 0.25 & 2 & $0.64 \pm 0.01$ & 0.098 & $0.5 \pm 0.1$ \\
\hline 0.05 & 2 & $0.85 \pm 0.01$ & 0.144 & $0.5 \pm 0.1$ & 0.30 & 2 & $0.56 \pm 0.01$ & 0.081 & " \\
\hline 0.10 & 2 & $0.82 \pm 0.02$ & 0.137 & $*$ & 0.35 & 2 & $0.47 \pm 0.01$ & 0.062 & " \\
\hline 0.15 & 2 & $0.77 \pm 0.02$ & 0.126 & " & 0.40 & 2 & $0.37 \pm 0.01$ & 0.042 & " \\
\hline 0.20 & 2 & $0.71 \pm 0.01$ & 0.113 & $*$ & 0.45 & 2 & $0.26 \pm 0.01$ & 0.023 & " \\
\hline
\end{tabular}

TABLE I. Critical couplings and exponents for on-site interactions

\begin{tabular}{|c|c|c|c|c|c|c|c|c|c|}
\hline \multicolumn{10}{|c|}{ the transition for } \\
\hline & \multicolumn{4}{|c|}{ off-diagonal LRO } & \multicolumn{5}{|c|}{ diagonal LRO } \\
\hline$n_{0}$ & $z$ & $K^{*}$ & $J^{*} / U_{0}$ & $\nu$ & $z$ & $K^{*}$ & $J^{*} / U_{0}$ & $\nu$ & $\beta$ \\
\hline 0.5 & 1 & $0.775 \pm 0.005$ & 0.127 & $0.65 \pm 0.08$ & 1 & $0.837 \pm 0.005$ & 0.141 & $0.55 \pm 0.05$ & $0.21 \pm 0.04$ \\
\hline 0.4 & 2 & $0.645 \pm 0.008$ & 0.099 & $0.44 \pm 0.08$ & 2 & $0.749 \pm 0.006$ & 0.122 & $0.5 \pm 0.1$ & $0.25 \pm 0.10$ \\
\hline 0.4 & & & & & 1 & $0.747 \pm 0.007$ & 0.121 & $0.55 \pm 0.06$ & $0.21 \pm 0.08$ \\
\hline 0.2 & 2 & $0.446 \pm 0.005$ & 0.057 & $0.5 \pm 0.1$ & \multicolumn{5}{|c|}{ for comparison: } \\
\hline 0.1 & 2 & $0.707 \pm 0.007$ & 0.112 & $0.49 \pm 0.11$ & \multicolumn{3}{|c|}{ mean-field: } & $1 / 2$ & $1 / 2$ \\
\hline 0.0 & 1 & $0.843 \pm 0.005$ & 0.142 & $0.61 \pm 0.08$ & \multicolumn{3}{|c|}{ 3D XY: } & $2 / 3$ & $1 / 3$ \\
\hline
\end{tabular}

TABLE II. Critical couplings and exponents for the different transitions with nearest-neighbor interactions $U_{1} / U_{0}=0.2$ 


\section{REFERENCES}

[1] B. G. Orr, H. M. Jaeger, A. M. Goldman, and C. G. Kuper, Phys. Rev. Lett. 56, 378 (1986); H. M. Jaeger, D. B. Haviland, B. G. Orr, and A. M. Goldman, Phys. Rev. B 40, 182 (1989); D. B. Haviland, Y. Liu, and A. M. Goldman, Phys. Rev. Lett. 62, 2180 (1989); Y. Liu, K. A. Greer, B. Nease, D. B. Haviland, G. Martinez, J. W. Haley, and A. M. Goldman, Phys. Rev. Lett. 67, 2068 (1991).

[2] A. F. Hebard and M. A. Paalanen, Phys. Rev. Lett. 65, 927 (1990).

[3] L. J. Geerligs, M. Peters, L. E. M. de Groot, A. Verbruggen, and J. E. Mooij, Phys. Rev. Lett. 63, 326 (1989); H. S. J. van der Zant, F. C. Fritschy, W. E. Elion, L. J. Geerligs, and J. E. Mooij, Phys. Rev. Lett. 69, 2971 (1992); H. S. J. van der Zant, L. J. Geerligs, and J. E. Mooij, Europhys. Lett. 19, 541 (1992); T. S. Tighe, M. T. Tuominen, J. M. Hergenrother, and M. Tinkham, Phys. Rev. B 47, 1145 (1993); P. Delsing, C. D. Chen, D. B. Haviland, Y. Harada, and T. Claeson, Phys. Rev. B 50, 3959 (1994); H. S. J. van der Zant, W. J. Elion, L. J. Geerligs, and J. E. Mooij, unpublished.

[4] K. B. Efetov, Sov. Phys. JETP 51, 1015 (1980).

[5] S. Doniach, Phys. Rev. B 24, 5063 (1981).

[6] M. P. A. Fisher, B. P. Weichman, G. Grinstein, and D. S. Fisher, Phys. Rev. B 40, 546 (1989).

[7] M. P. A. Fisher, Phys. Rev. Lett. 65, 923 (1990).

[8] G. T. Zimanyi in Strongly Correlated Electronic Materials, edited by K. S. Bedell et al. (Addison-Wesley, 1994).

[9] A. F. Andreev and I. M. Lifshitz, Sov. Phys. JETP 29, 1107 (1969).

[10] A. J. Leggett, Phys. Rev. Lett. 25, 1543 (1970).

[11] H. Matsuda and T. Tsuneto, Suppl. Prog. Theor. Phys 46, 411 (1970). 
[12] K. Liu and M. Fisher, J. Low. Temp. Phys. 10, 655 (1973).

[13] C. Bruder, R. Fazio, and G. Schön, Phys. Rev. B 47, 342 (1993); C. Bruder and G. Schön, in KT Transition and Superconducting Arrays, edited by D. Kim et al., (Min Eum Sa Co., Seoul, 1993), p. 175.

[14] E. Roddick and D. H. Stroud, Phys. Rev. B 48, 16600 (1993).

[15] M. W. Meisel, Physica 178, 121 (1992); and references therein.

[16] G. A. Lengua and J. M. Goodkind, J. Low. Temp. Phys. 79, 251 (1990).

[17] M. Gabay and A. Kapitulnik, Phys. Rev. Lett. 71, 2138 (1993); S. C. Zhang, Phys. Rev. Lett. 71, 2142 (1993).

[18] K. Mullen, H. T. C. Stoof, M. Wallin, and S. M. Girvin, Phys. Rev. Lett. 72, 4013 (1994).

[19] L. Balents, unpublished.

[20] G. G. Batrouni, R. T. Scalettar, G. T. Zimanyi, and A. P. Kampf, Phys. Rev. Lett. 74, 2527 (1995); A. P. Kampf, G. T. Zimanyi, G. G. Batrouni, and R. T. Scalettar, unpublished.

[21] E. Roddick and D. H. Stroud, Phys. Rev. B 51, 8672 (1995).

[22] D. R. Nelson, Phys. Rev. Lett. 60, 1973 (1988).

[23] M. P. A. Fisher and D-H. Lee, Phys. Rev. B 39, 2756 (1989).

[24] M. V. Feigel'man, V. B. Geshkenbein, L. B. Ioffe, and A. I. Larkin, Phys. Rev. B 48, 16641 (1993).

[25] G. Blatter, M. V. Feigel'man, V. B. Geshkenbein, A. I. Larkin, and V. M. Vinokur, Rev. Mod. Phys. 66, 1125 (1994).

[26] E. Frey, D. R. Nelson, and D. S. Fisher, Phys. Rev. B 49, 9723 (1994). 
[27] R. Baltin, K-H. Wagenblast, G. Schön, and A. van Otterlo, unpublished.

[28] A. van Otterlo and K-H. Wagenblast, Phys. Rev. Lett. 72, 3598 (1994).

[29] C. Bruder, R. Fazio, A. P. Kampf, A. van Otterlo, and G. Schön, Physica Scripta T 42, 159 (1992).

[30] K. Sheshadri, H. R. Krishnamurthy, R. Pandit, and T. V. Ramakrishnan, Europhys. Lett. 22, 257 (1993).

[31] N. D. Mermin and H. Wagner, Phys. Rev. Lett. 17, 1133 (1966); P. C. Hohenberg, Phys. Rev. 158, 383 (1967).

[32] K-H. Wagenblast, Diplomarbeit Universität Karlsruhe 1994, unpublished.

[33] M. Gutzwiller, Phys. Rev. Lett. 10, 159 (1963).

[34] D. A. Huse, and V. Elser, Phys. Rev. Lett. 60, 2531 (1988).

[35] D. S. Rokhsar, and B. G. Kotliar, Phys. Rev. B 44, 10328 (1991).

[36] W. Krauth, M. Caffarel, and J.-P. Bouchard, Phys. Rev. B 45, 3137 (1992).

[37] G. G. Batrouni, R. T. Scalettar, and G. T. Zimanyi, Phys. Rev. Lett. 65, 1765 (1990);

R. T. Scalettar, G. G. Batrouni, and G. T. Zimanyi, Phys. Rev. Lett. 66, 3144 (1991).

[38] P. Niyaz, C. Y. Fong, R. T. Scalettar, and G. G. Batrouni, Phys. Rev. B 50, 362 (1994);

P. Niyaz, R. T. Scalettar, C. Y. Fong, and G. G. Batrouni, Phys. Rev. B 44, 7143 (1991).

[39] W. Krauth and N. Trivedi, Europhys. Lett. 14, 627 (1991).

[40] E. S. Sørensen, M. Wallin, S. M. Girvin and A. P. Young, Phys. Rev. Lett 69, 828 (1992); M. Wallin, E. S. Sørensen, S. M. Girvin, and A. P. Young, Phys. Rev. B 49, 12115 (1994).

[41] J. K. Freericks and H. Monien, Europhys. Lett. 26, 545 (1994). 
[42] J. K. Freericks and H. Monien, unpublished.

[43] J. V. José, L. P. Kadanoff, S. Kirkpatrick, and D. R. Nelson, Phys. Rev. B 16, 1217 (1977); L. P. Kadanoff, J. Phys. A 11, 1399 (1978).

[44] J. Villain, J. Physique 36, 581 (1975).

[45] M.-C. Cha, M. P. A. Fisher, S. M. Girvin, M. Wallin, and A. P. Young, Phys. Rev. B 44, 6883 (1991); M. P. A. Fisher, G. Grinstein, and S. M. Girvin, Phys. Rev. Lett. 64, $587(1990)$.

[46] A. van Otterlo, K-H. Wagenblast, R. Fazio, and G. Schön, Phys. Rev. B 48, 3316 (1993).

[47] J. G. Kissner and U. Eckern, Z. Phys. B 91, 155 (1993).

[48] A. P. Kampf and G. T. Zimanyi, Phys. Rev. B 47, 279 (1993).

[49] L. Balents, and E. Frey, private communication. 


\section{FIGURES}

FIG. 1. : Phase diagram for hard-core bosons, as obtained from spin mean-field theory on the XXZ model. Nearest and next-nearest neighbor $\left(U_{2} / U_{1}=0.1\right)$ interactions are included. Several phases appear: superfluid (SF), Mott-insulating (MI), checkerboard solid (Sol) and the corresponding supersolid (SSol1), and quarter-filled (QF) solid with the corresponding supersolid (SSol2).

FIG. 2. : Phase diagrams for soft-core bosons, as obtained from the mean-field analysis of the QP model with on-site interaction $U_{0}$ only (round curves on the left). The symbols are the Monte Carlo data as discussed in Sect. \. The sharp lobes are results of the third-order $t / U$-expansion of Ref. [41] (extreme right) and the extrapolation to infinite order in $t / U$ from Ref. [42]. The superfluid phase is denoted by "SF" and the Mott-insulating phase by "MI".

FIG. 3. : Phase diagrams for soft-core bosons, as obtained from the mean-field analysis of the QP model with on-site $\left(U_{0}\right)$ and nearest-neighbor $\left(U_{1} / U_{0}=1 / 5\right)$ interaction. The symbols are the Monte Carlo data as discussed in Sect. \. The checkerboard charge-density wave is denoted by "Sol", the supersolid phase by "SSol", the superfluid phase is denoted by "SF" and the Mott-insulating phase by "MI".

FIG. 4. : Supersolid region "SSol" at $n_{0}=0.5$ as a function of $U_{1} / U_{0}$ in the mean-field approximation of Section [IIB. Inset: Occupation-number probability $\left|c_{2}\right|^{2}$ at $n_{0}=0.5$ for the two sublattices $A$ and $B$ at the particular value of $U_{1} / U_{0}=0.2$.

FIG. 5. : Mean-field phase diagram for nearest-neighbor and next-nearest neighbor interactions, $U_{1} / U_{0}=0.2, U_{2} / U_{0}=0.02$. In addition to the phase diagram in Fig. 3, small lobes at quarter filling and tree-quarter filling appear at small $J$ with their corresponding supersolids. The phases are labeled as in Fig. 1.

FIG. 6. : Phase diagram for the Quantum-Phase model as determined from the variational Ansatz with nearest-neighbor interactions, $U_{1} / U_{0}=1 / 5$. 
FIG. 7. : Phase diagram for the Bose-Hubbard model as determined from the variational Ansatz with nearest-neighbor interactions, $U_{1} / U_{0}=1 / 5$. For small $n_{0}$ the supersolid phase vanishes at point $\alpha$.

FIG. 8. : Scaling plot of the Monte Carlo data for the superfluid stiffness $\rho_{0}$ for $n_{0}=0$ with on-site interaction only.

FIG. 9. : Monte Carlo data for the superfluid stiffness $\rho_{0}$ and the structure factor $S_{\pi}$ for $n_{0}=0.5$ with nearest-neighbor interaction $U_{1} / U_{0}=1 / 5$. We clearly observe two distinct transitions at the crossing points of these scaling plots, and a coexistence phase in between.

FIG. 10. : Scaling plots for the structure factor $S_{\pi}$ for $z=1$ (lower curve) and $z=2$ (upper curve) at $n_{0}=0.4$ and $U_{1} / U_{0}=1 / 5$. 
Fig. 1:

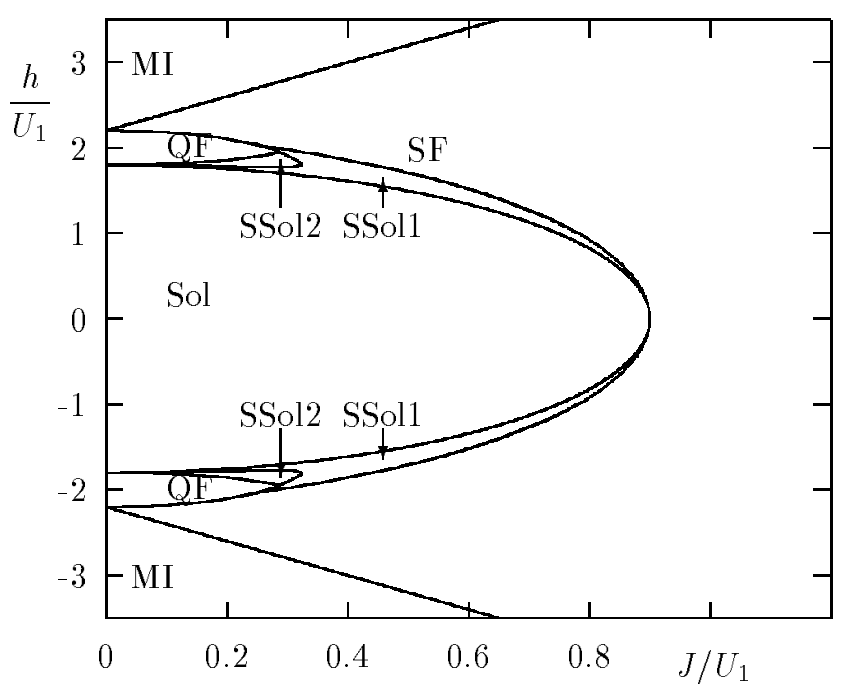

Fig. 2:

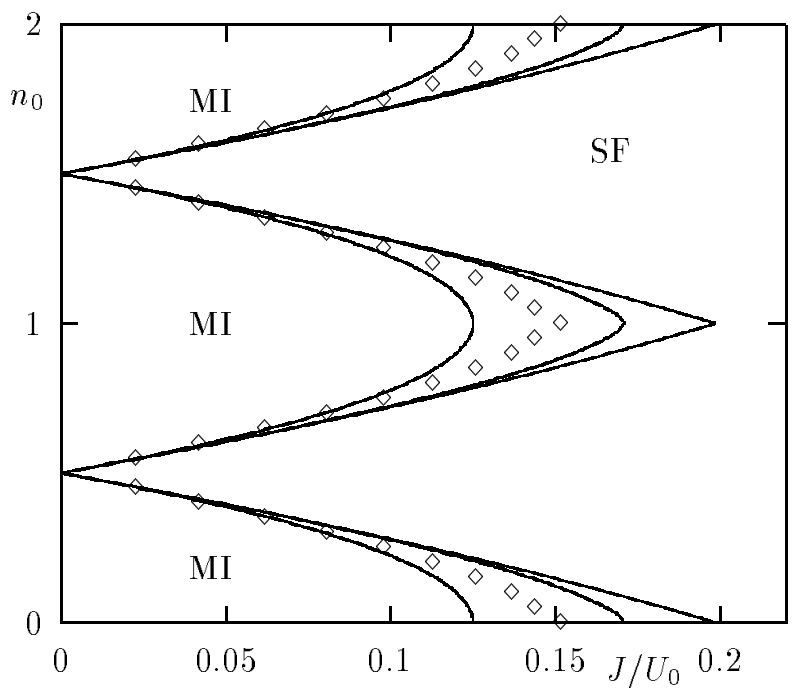


Fig. 3:

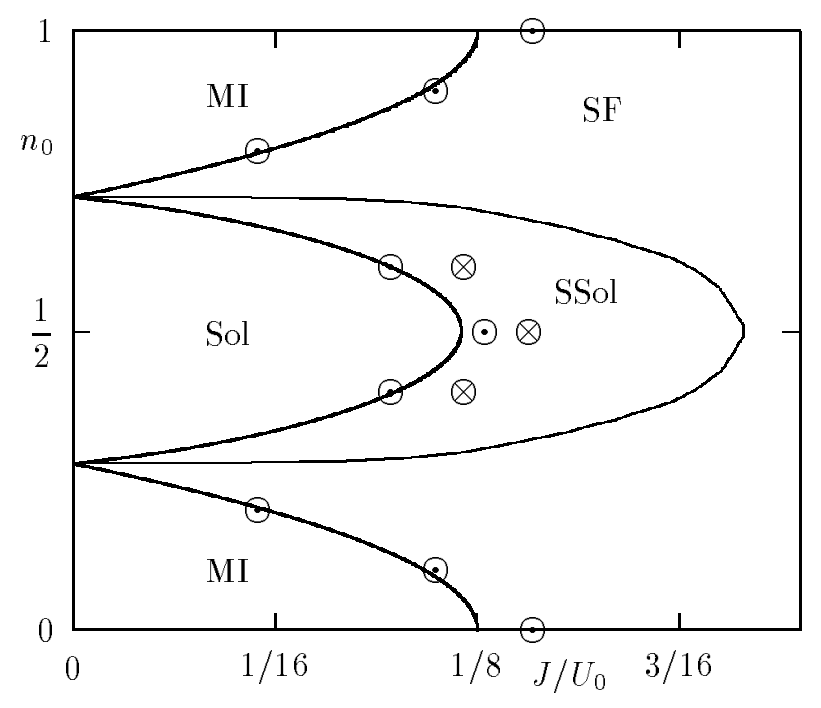

Fig. 4:

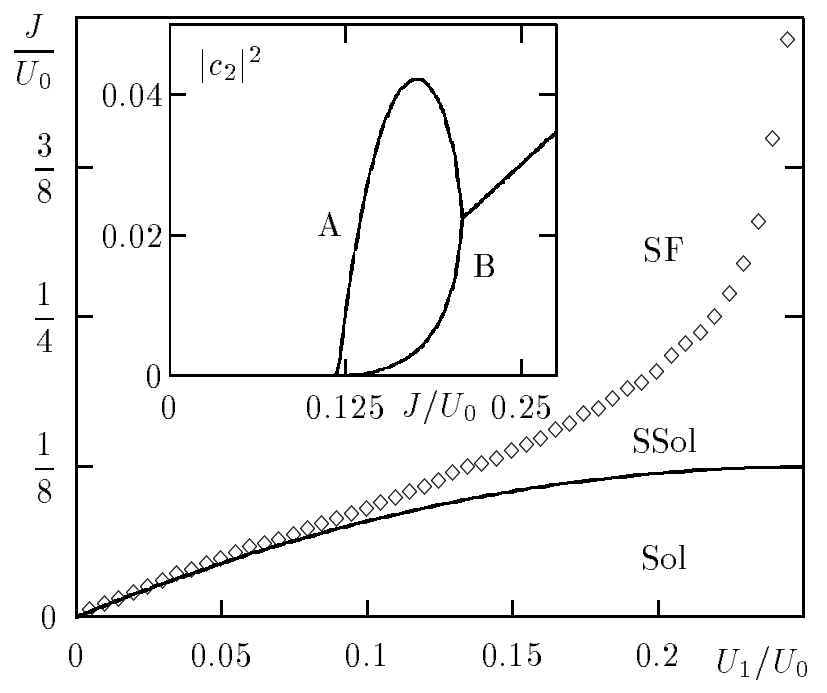


Fig. 5:

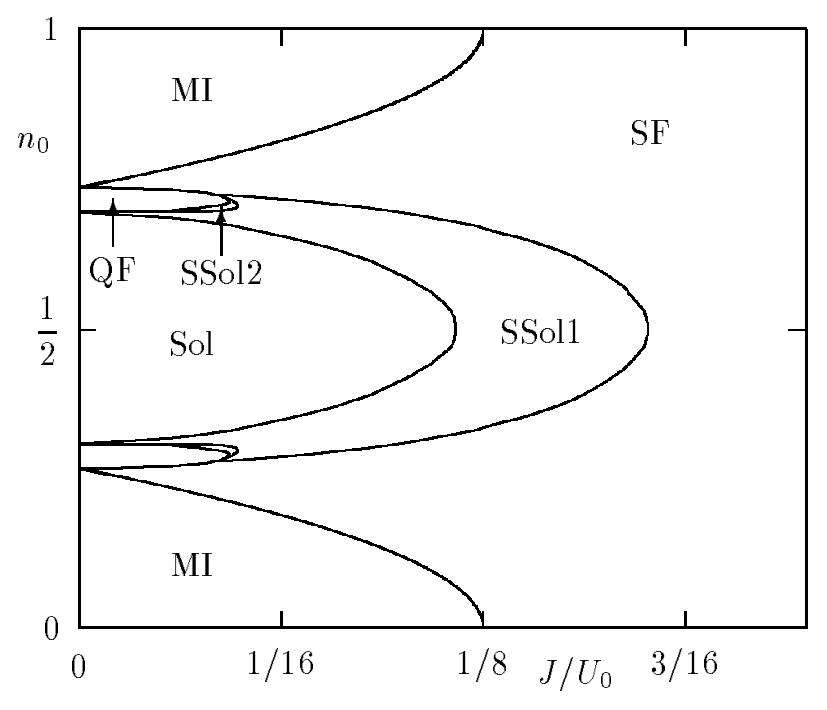

Fig. 6:

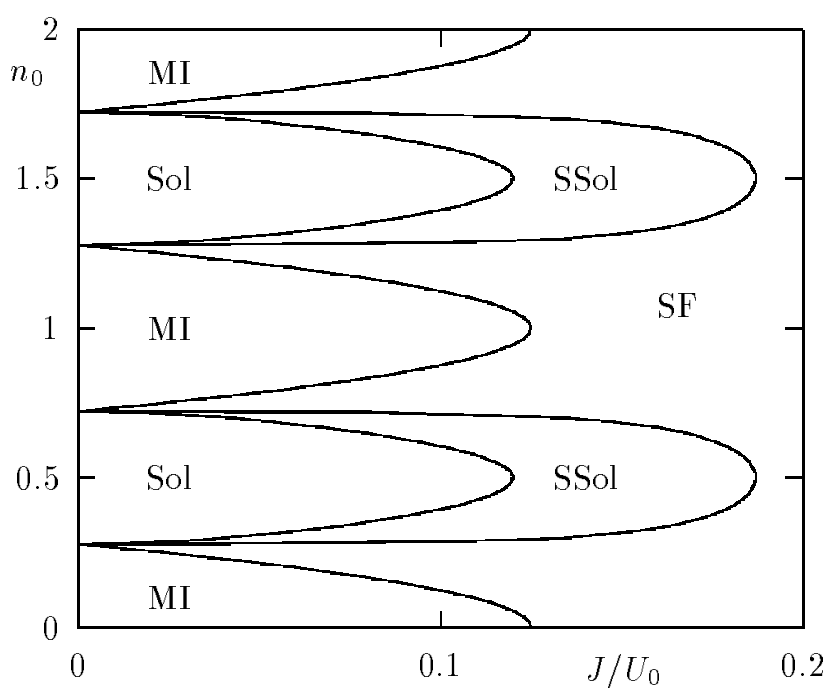


Fig. 7:

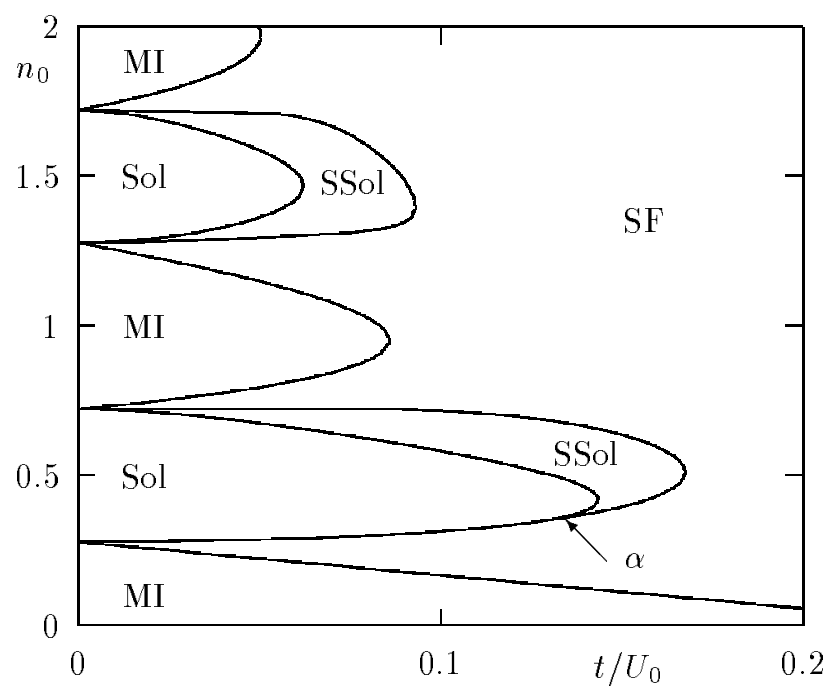

Fig. 8:

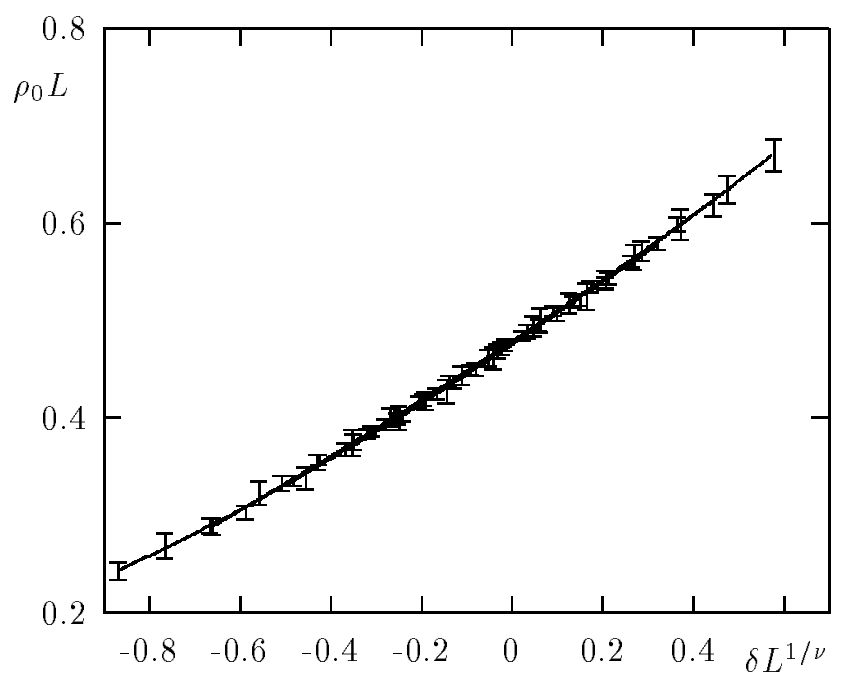


Fig. 9:

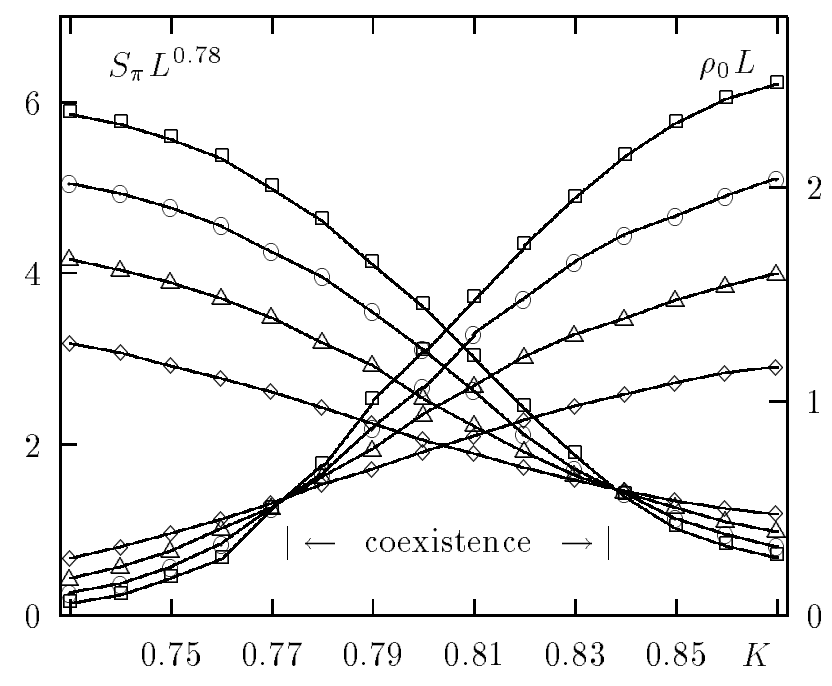

Fig. 10:

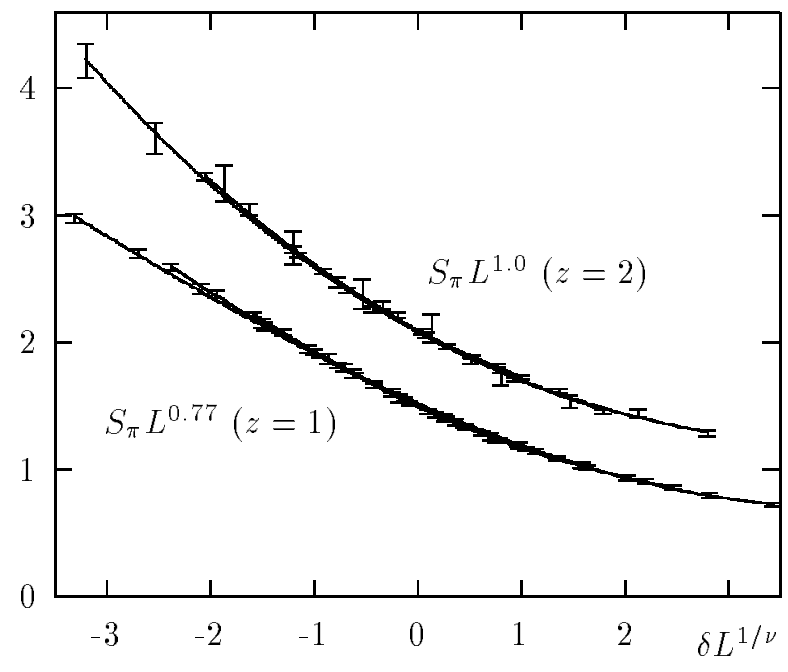

